TI 2013-093/II

Tinbergen Institute Discussion Paper
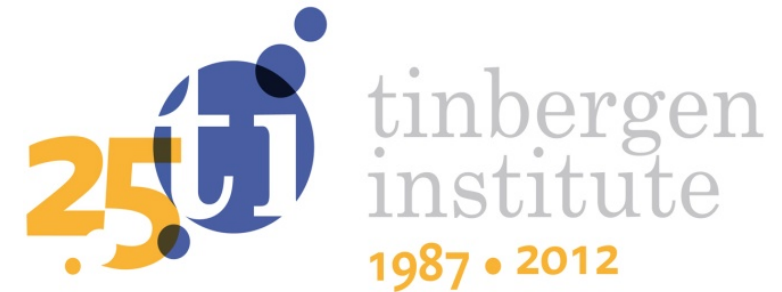

\title{
Order Monotonic Solutions for Generalized Characteristic Functions
}

\author{
René van den Brink ${ }^{1}$ \\ Enrique González-Aranguena² \\ Conrado Manue/2 \\ Mónica del Pozo 3
}

\footnotetext{
1 Faculty of Economics and Business Administration, VU University Amsterdam, and Tinbergen Institute;

2 Facultad de Estudios Estadísticos, Universidad Complutense de Madrid, Spain;

3 Universidad Carlos III de Madrid, Spain.
} 
Tinbergen Institute is the graduate school and research institute in economics of Erasmus University Rotterdam, the University of Amsterdam and VU University Amsterdam.

More TI discussion papers can be downloaded at http://www.tinbergen.nl

Tinbergen Institute has two locations:

Tinbergen Institute Amsterdam

Gustav Mahlerplein 117

1082 MS Amsterdam

The Netherlands

Tel.: +31(0)205251600

Tinbergen Institute Rotterdam

Burg. Oudlaan 50

3062 PA Rotterdam

The Netherlands

Tel.: +31(0)10 4088900

Fax: $+31(0) 104089031$

Duisenberg school of finance is a collaboration of the Dutch financial sector and universities, with the ambition to support innovative research and offer top quality academic education in core areas of finance.

DSF research papers can be downloaded at: http://www.dsf.nl/

Duisenberg school of finance

Gustav Mahlerplein 117

1082 MS Amsterdam

The Netherlands

Tel.: +31(0)20 5258579 


\title{
Order Monotonic Solutions for Generalized Characteristic Functions
}

\author{
René van den Brink*Enrique González-Aranguena ${ }^{\ddagger}$ Conrado ManuelMónica del Pozo ${ }^{\ddagger}$
}

July 17, 2013

\begin{abstract}
Generalized characteristic functions extend characteristic functions of 'classical' TU-games by assigning a real number to every ordered coalition being a permutation of any subset of the player set. Such generalized characteristic functions can be applied when the earnings or costs of cooperation among a set of players depends on the order in which the players enter a coalition.

In the literature, the two main solutions for generalized characteristic functions are the one of Nowak and Radzik (1994), shortly called NR-value, and the one introduced by Sánchez and Bergantiños (1997), shortly called SB-value. In this paper, we introduce the axiom of order monotonicity with respect to the order of the players in a unanimity coalition, requiring that players who enter earlier should get not more in the corresponding (ordered) unanimity game than players who enter later. We propose several classes of order monotonic solutions for generalized characteristic functions that contain the NR-value and SB-value as special (extreme) cases. We also provide axiomatizations of these classes.
\end{abstract}

Keywords: Cooperative TU-game, generalized characteristic function, order monotonicity JEL code: $\mathrm{C} 71$

AMS subject classification:

\footnotetext{
* Department of Econometrics and Tinbergen Institute, VU University, De Boelelaan 1105, 1081 HV Amsterdam, The Netherlands. e-mail: jrbrink@feweb.vu.nl

${ }^{\ddagger}$ Dpto. de Estadística e I.O. III. Facultad de Estudios Estadísticos, Universidad Complutense de Madrid, Avda. Puerta de Hierro s/n, 28040-Madrid (Spain) (e-mail: egaran@estad.ucm.es, conrado@estad.ucm.es)

${ }^{\S}$ Dpto. de Economía, Universidad Carlos III de Madrid (Spain) (e-mail: mopozoj@eco.uc3m.es)
} 


\section{Introduction}

Generalized characteristic functions on a player set $N$, introduced by Nowak and Radzik (1994), extend characteristic functions of 'classical' TU-games by assigning a real number to every ordered coalition of $N$. Here an ordered coalition of $N$ is a permutation of any subset of $N$. Classical characteristic functions form the special class where the real number or worth assigned to any ordered coalition only depends on the players that are part of this ordered coalition, i.e. it does not matter in which order the players enter the coalition. Generalized characteristic functions can be used in situations where the worth (or cost) that can be generated by a set of players depends on the order in which the players enter.

Consider, for example, the airport games of Littlechild and Owen (1973) to allocate the building and maintenance costs of airport landing strips, see also Littlechild and Thompson (1977). An airport cost situation consists of a set of airplanes (being the players in the game) and for each airplane a nonnegative cost of the airline strip that is necessary for this airplane to land. Since the airplanes are different they need landing strips of different length. In the associated airport game, the worth of a coalition (being a subset of the set of airplanes $N$ ) is the cost of the airline strip needed for the largest airplane in this coalition (assuming that larger airplanes need longer and more expensive landing strips). ${ }^{1}$ But this means that building a landing strip of a certain size does not depend on the order in which the airplanes enter the coalition. The worth (cost) of a coalition is always fully determined by the cost for the largest airplane in the coalition. However, in real life construction industry it is usually more expensive to build a project in several steps than to build it fully at once. For example, when one wants to extend an existing landing strip then all the machinery has to be brought back to the airport, everything needs to be setup again, maybe some reconstruction or preparation needs to be done before being able to extend the existing landing strip. Then it would have been less costly to have built the longer landing strip at once. Therefore, instead of modelling an airport cost problem on $n$ airplanes by an $n$ dimensional cost vector, it seems more realistic to model it by an $n \times n$ dimensional cost matrix, which $i j^{t h}$ component $c_{i j}, i \in\{1, \ldots, n\}, j \in\{2, \ldots, n\}$, is the cost of building (extending) an airline strip suitable for airplane $i$ when the landing strip is built already for airplane $j-1$ (and smaller airplanes), and the first column gives the cost for building the airline strip for $i$ when there is nothing built yet. So, we might consider the first column as a standard airport cost problem.

Example 1.1 Suppose that there are three airplanes $N=\{1,2,3\}$, where the costs of building an airline strip for airplane $i \in N$ is $c=\left(c_{i}\right)_{i \in N}=(1,3,4)$. However, it can be that extending the airline strip for airplane 1 to one for airplane 2 costs 3 additional to the cost made to build the already existing airline strip. If, further extending the airline strip for airplane 1 to one for airplane 3 costs 3 , and extending the airline strip for airplane 2 to one for airplane 3 costs 2 , this

\footnotetext{
${ }^{1}$ Instead of airplanes, in an airport game every airplane landing is a player but for convenience we simply call these airplanes.
} 
can be represented by cost matrix

$$
c=\left[\begin{array}{lll}
1 & 0 & 0 \\
3 & 3 & 0 \\
4 & 3 & 2
\end{array}\right]
$$

Such an airport cost problem, where the cost of the landing strip depends on the order in which airplanes announce that they want to use the landing strip, cannot be modelled by a classical TU-game. Although the entrance of an airplane that is not larger than the largest airplane in the coalition does not change the worth, when an airplane larger than the largest airplane in the coalition enters, then the additional cost depends on which is the largest airplane in the already existing coalition. Such generalized airport cost problems can be modeled by a generalized characteristic function where the worth of an ordered coalition of airplanes is the cost of the landing strip if it would be built sequentially, in each step being large enough to allow the corresponding airplane to land.

In the literature, the two main solutions for generalized characteristic functions are the one of Nowak and Radzik (1994), which we will call the NR-value, and the one introduced by Sánchez and Bergantiños (1997), which we refer to as the SB-value. Both solutions extend the Shapley value of classical TU-games in the sense that for a generalized characteristic function that represents a classical characteristic function they yield the Shapley value of that classical game.

Nowak and Radzik (1994) characterize their solution by efficiency, linearity and some null player property, where the last property states that players who always contribute zero when they enter an ordered coalition as last player, should get a zero payoff. Sánchez and Bergantiños (1997) criticize this null player property by arguing that in this definition a player is a null player even when it contributes to an ordered coalition in the sense that without participation of this player the coalition loses its worth. Instead of only considering the contributions to ordered coalitions when a player enters as last, Sánchez and Bergantiños (1997) consider the effect on the worth of a coalition of a player leaving in any position in the ordered coalition. If for any position in any ordered coalition this loss is zero, then Sánchez and Bergantiños (1997) call this a null player, and in their corresponding null player property such a player should get a zero payoff. Since this null player property is weaker than that of Nowak and Radzik (1994), Sánchez and Bergantiños (1997) added a symmetry axiom, requiring that players who are symmetric earn the same payoff, where two players are called symmetric if for every ordered coalition not containing both of them they add the same contribution when entering at the same position. They characterized their solution by efficiency, linearity, their (weak) null player property and symmetry.

Although we agree with the critique of Sánchez and Bergantiños (1997) on the null player property of Nowak and Radzik (1994), we further criticize their symmetry property. The definition of a symmetric player allows that two players $i$ and $j$ are symmetric, when for every permutation 
of any coalition not containing these two players, the marginal contribution of these players to any position in that permutation is the same, but for any permutation of any coalition containing these two players, exchanging their position yields a different worth.

Therefore, we introduce the axiom of order monotonicity with respect to the order of the players in a unanimity coalition, requiring that players who enter earlier should get not more in the corresponding unanimity game than players who enter later. This is weaker than symmetry since in a unanimity game all players in the unanimity coalition are symmetric and therefore earn the same payoff if symmetry is satisfied. Together with efficiency, linearity, the null player property (of Sánchez and Bergantiños (1997)), anonymity and nonnegativity, this order monotonicity characterizes a class of solutions, that is defined using games with a permission structure as introduced in Gilles, Owen and van den Brink (1992), Gilles and Owen (1999), van den Brink and Gilles (1996) and van den Brink (1997), containing the NR-value and SB-value as extreme cases.

After introducing and characterizing this class, we give special attention to two subclasses of these solutions, both containing the solutions of Nowak and Radzik (1994) and Sánchez and Bergantiños (1997) as extreme cases. To motivate the first class note that, under efficiency, the symmetry axiom of Sánchez and Bergantiños (1997) is incompatible with the null player property of Nowak and Radzik (1997) since according to the last axiom, in the unanimity game on an ordered coalition all players except the last one in this (ordered) coalition should get zero, implying that the last player in the ordered unanimity coalition should get all the worth under efficiency, while according to the symmetry axiom all players in the coalition should get the same. We define compatible pairs of null player and symmetry axioms that, together with efficieny and linearity, characterize a solution.

The second class is the so-called family of geometric solutions that are used in del Pozo, Manuel, González-Arangüena and Owen (2011), to define centrality measures for directed networks. We characterize this class by extending and modifying the enemy players property and null contributions for indifferent players, used by Manuel, González-Arangüena and van den Brink (2013) to axiomatize the Shapley value for classical TU-games.

The paper is organized as follows. Section 2 contains preliminaries on games in generalized characteristic function and games with a permission structure. In Section 3 we discuss order monotonicity of solutions for games in generalized characteristic functions. In Sections 4 and 5 we discuss the two special classes of order monotonic solutions mentioned above.

\section{Preliminaries}

\subsection{Games and Generalized Games}

A situation in which a finite set of players $N \subset \mathbb{N}$ can generate certain payoffs by cooperation can be described by a cooperative game in characteristic function form (also known as cooperative game with transferable utility or simply TU-game) being a pair $(N, \hat{v})$ where the characteristic 
function $\hat{v}: 2^{N} \rightarrow \mathbb{R}$ is a real function defined on $2^{N}$ (the set of all subsets of $N$ ), that satisfies $\hat{v}(\emptyset)=0$. For each coalition $S \in 2^{N}$, the worth $\hat{v}(S)$ represents the (transferable) utility that players in $S$ can obtain if they decide to cooperate. When there is no ambiguity with respect to the players set $N$, we will identify the game $(N, \hat{v})$ with its characteristic function $\hat{v}$. In the sequel we will denote the cardinality of coalitions $S, T, R \in 2^{N}$ by lower case $s, t, r$. We will denote by $G^{N}$ the set of all characteristic functions with player set $N$. It is well-known that $G^{N}$ is a $2^{n}-1$ dimensional vector space, $n=|N|$, with the unanimity games $\left\{\hat{u}_{S}\right\}_{\emptyset \neq S \subset N}$ as basis. For every $S \subseteq N, S \neq \emptyset$, the unanimity game $\hat{u}_{S}$ is defined by $\hat{u}_{S}(T)=1$ if $S \subseteq T$, and $\hat{u}_{S}(T)=0$, otherwise. For a given $\hat{v} \in G^{N}$, the unanimity coefficients (i.e. the coordinates of $\hat{v}$ in the unanimity basis) $\left\{\Delta_{\hat{v}}(T)\right\}_{\emptyset \neq T \subset N}$ are given by (see Harsanyi (1959))

$$
\Delta_{\hat{v}}(T)=\sum_{R \subseteq T}(-1)^{t-r} \hat{v}(R), \emptyset \neq T \subseteq N
$$

A solution or value for TU-games is a function which assigns a payoff vector $x \in \mathbb{R}^{N}$ to every TU-game in $G^{N}$. One of the most famous solutions is the Shapley value (Shapley, 1953), $\varphi^{S h}$, which is given by:

$$
\varphi_{i}^{S h}(N, \hat{v})=\sum_{S \subseteq N \backslash\{i\}} \frac{(n-s-1) ! s !}{n !}(\hat{v}(S \cup\{i\})-\hat{v}(S)), \quad \text { for all } i \in N .
$$

Nowak and Radzik (1994) introduced the concept of game in generalized characteristic function form where the order in which a coalition is formed influences the worth that can be generated. For each $S \in 2^{N} \backslash\{\emptyset\}$, let $\Pi(S)$ denote the set of all permutations or ordered coalitions of the players in $S$ and, for notational convenience, $\Pi(\emptyset)=\{\emptyset\}$. We denote $\Omega(N)=$ $\{T \in \Pi(S) \mid S \subseteq N\}$ as the set of all ordered coalitions with players in $N$. A game in generalized characteristic function form is a pair $(N, v), N$ being the player set and $v: \Omega(N) \rightarrow \mathbb{R}$ a real function (the generalized characteristic function), defined on $\Omega(N)$ and satisfying $v(\emptyset)=0$.

For each $S \subseteq N$, and for every ordered coalition $T \in \Pi(S), v(T)$ represents the economic possibilities of the players in $S$ if the coalition is formed following the order given by $T$.

Example 2.1 Consider the generalized airport cost problem of Example 1.1. The corresponding generalized characteristic function is $v(1)=1, v(2)=v(21)=3, v(3)=v(31)=v(32)=$ $v(312)=v(321)=4, v(12)=1+3=4, v(13)=v(132)=1+3=4, v(23)=v(213)=v(231)=$ $3+2=5$ and $v(123)=6$.

We denote by $\mathcal{G}^{N}$ the set of all generalized characteristic functions with player set $N$, and $\mathcal{G}=\left\{(N, v) \mid N \subset \mathbb{N}, v \in \mathcal{G}^{N}\right\}$. As in the case of games in $G^{N}$, we will sometimes identify the game with its characteristic function.

Given an ordered coalition $T \in \Omega(N)$, there exists $S \subseteq N$ such that $T \in \Pi(S)$. We will denote by $H(T)=S$ the set of players in the ordered coalition $T$, and $t=|H(T)|$. Each ordered coalition $T=\left(i_{1}, \ldots, i_{t}\right) \in \Omega(N)$ establishes a strict linear order $\prec_{T}$ in $H(T)$, defined as follows: 
for all $i, j \in H(T), i \prec_{T} j$ ( $i$ precedes $j$ in $T$ ) if and only if there exist $k, l \in\{1, \ldots, t\}, k<l$, such that $i=i_{k}, j=i_{l}$.

Given two ordered coalitions $T, R \in \Omega(N)$, we denote $T \widetilde{\subseteq} R$ if and only if:

a) $H(T) \subseteq H(R)$, and

b) $\forall i, j \in H(T), i \prec_{T} j$ implies $i \prec_{R} j$.

It is known that $\mathcal{G}^{N}$ is a vector space with dimension $|\Omega(N)|-1$ where the generalized unanimity games $\left\{w_{T}\right\}_{\emptyset \neq T \in \Omega(N)}$ form a basis of $\mathcal{G}^{N}$. For every $T \in \Omega(N)$, the generalized unanimity game $w_{T}$ is defined by $w_{T}(R)=1$ if $T \widetilde{\subset} R$, and $w_{T}(R)=0$ otherwise.

For a given $v \in \mathcal{G}^{N}$, Sánchez and Bergantiños (1997) proved that the generalized unanimity coefficients (i.e. the coordinates of $v$ in the generalized unanimity basis) $\left\{\Delta_{v}^{*}(T)\right\}_{\emptyset \neq T \in \Omega(N)}$ are given by

$$
\Delta_{v}^{*}(T)=\sum_{R \widetilde{\subset} T}(-1)^{t-r} v(R), T \in \Omega(N) \backslash\{\emptyset\} .
$$

Observe that there exists an isomorphism between the vector space $G^{N}$ and the subspace of $\mathcal{G}^{N}$ consisting of all games for which $v(T)=v(R)$ if $H(T)=H(R)$ holds, i.e. the order in which the coalitions are formed is irrelevant. For $v \in \mathcal{G}^{N}$ and $S \subset N$ we define the subgame $\left(S,\left.v\right|_{S}\right)$ by $\left.v\right|_{S}(T)=v(T)$ for all $T \in \Omega(S)$.

For ordered coalition $T \in \Omega(N)$ let $j(T) \in\{1, \ldots, t\}$ be the position of player $j \in H(T)$ in $T$. So, for $T=\left(i_{1}, i_{2}, \ldots, i_{t}\right)$ we have $i_{k}(T)=k$ for all $k \in\{1, \ldots, t\}$. For player $i \in N \backslash H(T)$, let $\left(T, i^{l}\right), l \in\{1, \ldots, t+1\}$, be the ordered coalition that is obtained from $T$ by putting $i$ on the $l^{\text {th }}$ position and moving all players on position $l$ or higher one position backwards. So, $i\left(T, i^{l}\right)=l, j\left(T, i^{l}\right)=j(T)$ for all $j \in H(T)$ with $j(T) \in\{1, \ldots, l-1\}$, and $j\left(T, i^{l}\right)=j(T)+1$ for all $j \in H(T)$ with $j(T) \in\{l, \ldots, t\}$.

A solution for generalized characteristic functions is a function defined on $\mathcal{G}$ that assigns to every $(N, v) \in \mathcal{G}$ a vector in $\mathbb{R}^{N}$. In their seminal paper, Nowak and Radzik (1994) define and characterize a solution for generalized characteristic functions. This solution, which we will refer to as the Nowak-Radzik value, or shortly $N R$-value, is the solution $\psi^{N R}$ assigning to every generalized characteristic function $(N, v)$ the payoffs

$$
\psi_{i}^{N R}(N, v)=\sum_{T \in \Omega(N \backslash\{i\})} \frac{(n-t-1) !}{n !}\left(v\left(T, i^{t+1}\right)-v(T)\right) \text {, for all } i \in N .
$$

An alternative expression for this solution (based on the generalized unanimity coefficients of $v$ ) is:

$$
\psi_{i}^{N R}(N, v)=\sum_{\substack{T \in \Omega(N) \\ i(T)=t}} \frac{\Delta_{v}^{*}(T)}{t !}, \text { for all } i \in N
$$

Later, Sánchez and Bergantiños (1997) define and study another solution for generalized characteristic functions, which we call the Sánchez-Bergantiños value or shortly SB-value. This 
solution assigns to every generalized characteristic function the payoffs $\psi^{S B}(N, v)$ given by

$$
\psi_{i}^{S B}(N, v)=\sum_{T \in \Omega(N \backslash\{i\})} \frac{(n-t-1) !}{n !(t+1)} \sum_{l=1}^{t+1}\left(v\left(T, i^{l}\right)-v(T)\right), \text { for all } i \in N .
$$

An alternative expression (based on the generalized unanimity coefficients of $v$ ) is:

$$
\psi_{i}^{S B}(N, v)=\sum_{\substack{T \in \Omega(N) \\ i \in H(T)}} \frac{1}{t ! t} \Delta_{v}^{*}(T) \text { for all } i \in N .
$$

Nowak and Radzik (1994) axiomatize their value by the following three axioms. First, efficiency states that the sum of the payoffs allocated over the players should be equal to the average worth of the 'grand coalition' $N$ when every order or permutation in which $N$ is formed is equally likely.

Axiom 2.2 (Efficiency) For every $(N, v) \in \mathcal{G}$, it holds that $\sum_{i \in N} \psi_{i}(N, v)=\frac{1}{n !} \sum_{T \in \Pi(N)} v(T)$.

Second, linearity is standard.

Axiom 2.3 (Linearity) For every $(N, v),(N, w) \in \mathcal{G}$ and $a, b \in \mathbb{R}$ it holds that $\psi(N$, av $+b w)=$ $a \psi(N, v)+b \psi(N, w)$, where $(N, a v+b w) \in \mathcal{G}$ is given by $(a v+b w)(T)=a \cdot v(T)+b \cdot w(T)$ for all $T \in \Omega(N)$.

Third, a player is a NR-null player in generalized characteristic function $(N, v) \in \mathcal{G}$ if it always adds zero to any ordered coalition when it enters last in the coalition ${ }^{2}$, i.e. if $v\left(T, i^{t+1}\right)=$ $v(T)$ for all $T \in \Omega(N \backslash\{i\})$.

Axiom 2.4 (NR-null player property) If $i \in N$ is a $N R$-null player in $(N, v) \in \mathcal{G}$ then $\psi_{i}(N, v)=0$.

Nowak and Radzik (1994) show that their value is the unique solution satisfying these three axioms.

Theorem 2.5 [Nowak and Radzik (1994), Theorem 1] A solution $\psi$ satisfies efficiency, linearity and the NR-null player property if and only if $\psi=\psi^{N R}$.

Sánchez and Bergantiños (1997) criticize the NR-null player property by arguing that a player is a null player even when it contributes to an ordered coalition in the sense that without participation of this player the coalition loses its worth. Instead of only considering the contributions to a coalition when a player enters as last one, Sánchez and Bergantiños (1997) consider the effect on the worth of a coalition of a player leaving in any position in the ordered coalition.

\footnotetext{
${ }^{2}$ Nowak and Radzik (1994) call such a player simply a null player, but since we will later use the null player notion of Sánchez and Bergantiños (1997), we call this a NR-null player.
} 
If for any position in any ordered coalition this loss is zero, then Sánchez and Bergantiños (1997) call this a null player. Since we will use this weaker notion of a null player, we say the player $i$ in $N$ is a null player in $(N, v) \in \mathcal{G}$ if $v\left(T, i^{l}\right)=v(T)$ for all $T \in \Omega(N \backslash\{i\})$ and $l \in\{1, \ldots, t+1\}$. The null player property then states that such a player earns a zero payoff.

Axiom 2.6 (Null player property) If $i \in N$ is a null player in $(N, v) \in \mathcal{G}$ then $\psi_{i}(N, v)=0$.

Since this null player property is weaker than the NR-null player property, Sánchez and Bergantiños (1997) added a symmetry axiom, requiring that symmetric players earn the same payoff. Players $i, j \in N$ are symmetric in $(N, v) \in \mathcal{G}$ if $v\left(T, i^{l}\right)=v\left(T, j^{l}\right)$ for all $T \in \Omega(N \backslash\{i, j\})$ and $l \in\{1, \ldots, t+1\}$.

Axiom 2.7 (Symmetry) If $i, j \in N$ are symmetric players in $(N, v) \in \mathcal{G}$ then $\psi_{i}(N, v)=$ $\psi_{j}(N, v)$.

Theorem 2.8 [Sánchez and Bergantiños (1997), Theorem 1] A solution $\psi$ satisfies efficiency, linearity, the null player property and symmetry if and only if $\psi=\psi^{S B}$.

In Section 3 we will further criticize the symmetry axiom.

\subsection{Games with a permission structure}

In a (cooperative) game with a permission structure, see Gilles, Owen and van den Brink (1992), van den Brink and Gilles (1996), Gilles and Owen (1999) and van den Brink (1997), it is assumed that players who participate in a cooperative TU-game (in characteristic function) are part of a hierarchical organization in which there are players that need permission or approval from certain other players before they are allowed to cooperate. For a finite set of players $N$ such a hierarchical organization is represented by a directed graph $(N, D)$ with $D \subseteq N \times N$, referred to as a permission structure on $N$. The directed links $(i, j) \in D$ are called arcs. The players in $F_{D}(i):=\{j \in N \mid(i, j) \in D\}$ are called the followers or successors of player $i$, while the players in $P_{D}(i):=\{j \in N \mid(j, i) \in D\}$ are called the predecessors of $i$ in $D$.

In the conjunctive approach as developed in Gilles, Owen and van den Brink (1992) and van den Brink and Gilles (1996), it is assumed that each player needs permission from all its predecessors before it is allowed to cooperate. This implies that a coalition $S \subseteq N$ is feasible if and only if for every player in the coalition it holds that all its predecessors belong to the coalition. The set of feasible coalitions in this approach thus is given by

$$
\Phi_{D}^{c}:=\left\{S \subseteq N \mid P_{D}(i) \subset S \text { for all } i \in S\right\}
$$

A triple $(N, \hat{v}, D)$ with $\hat{v}: 2^{N} \rightarrow \mathbb{R}$ such that $v(\emptyset)=0$, and $(N, D)$ a digraph is called a game with a permission structure. An approach using restricted games similar to the approach of Myerson (1977) for communication graph games assigns to every coalition in a game with a permission 
structure the worth of its largest feasible subset. ${ }^{3}$ Given a game with a permission structure $(N, \hat{v}, D)$, the conjunctive restriction of $\hat{v}$ on $D$ is the characteristic function $r_{N, \hat{v}, D}^{c}: 2^{N} \rightarrow \mathbb{R}$ given by $r_{N, \hat{v}, D}^{c}(S)=\hat{v}\left(\sigma_{N, D}^{c}(S)\right)$ for all $S \subseteq N$, where $\sigma_{N, D}^{c}(S)=\cup\left\{T \in \Phi_{N, D}^{c} \mid T \subseteq S\right\}$ is the largest conjunctive feasible subset of $S$ refered to as its conjunctive sovereign part. The conjunctive permission value $\varphi$ is the solution that assigns to every game with a permission structure the Shapley value of the conjunctive restricted game, i.e. $\varphi(N, \hat{v}, D)=\varphi^{S h}\left(N, r_{N, \hat{v}, D}^{c}\right)$.

A special class of games with a permission structure are those $(N, \hat{v}, D)$ where $\hat{v}$ is an additive game (i.e. $\hat{v}(S)=\sum_{i \in S} \hat{v}(\{i\})$ for all $\left.S \subseteq N\right)$, and $(N, D)$ is a line graph meaning that there is a permutation $\pi$ of the players such that $D=\{(\pi(i), \pi(i+1)) \mid i \in\{1, \ldots, n-1\}\}$. These games are also known as line-graph peer group games, see Brânzei, Fragnelli and Tijs (2002). ${ }^{4}$

\section{Order monotonic solutions}

Although we agree with the critique of Sánchez and Bergantiños (1997) on the null player property of Nowak and Radzik (1994), we further criticize their symmetry property. The definition of a symmetric player allows that two players $i$ and $j$ are symmetric, when for every ordered coalition not containing these two players, the marginal contribution of these players to any position in that order is the same, but for any ordered coalition containing these two players, exchanging their position yields a different worth. For example, considering the unanimity game of the order $(1,2,3)$ on $N=\{1,2,3\}$, according to the definition of Sánchez and Bergantiños (1997) all three players are symmetric, and therefore the symmetry axiom requires them to get the same payoff. Note that the null player property of Nowak and Radzik (1994) states that players 1 and 2 are null players and therefore earn zero, so all payoff goes to player 3 .

We consider both allocations to be extreme. We agree with Sánchez and Bergantiños (1997) that players 1 and 2, although being null players in the sense that their marginal contribution as last player to any ordered coalition is zero, they are still needed to earn a positive worth. Player 3 on its own earns only zero. ${ }^{5}$ However, we agree with Nowak and Radzik (1994) that player 3 should be rewarded for being the only player having a positive marginal contribution when entering, and therefore we propose a class of solutions which to this unanimity game assigns payoffs such that player 3 earns at least as much as player 2 who earns at least as much as player 1. We define this class of solutions using cooperative games with a permission structure, in particular with a line-graph structure.

Given ordered coalition $T=\left(i_{1}, \ldots, i_{t}\right) \in \Omega(N)$, define the directed line-graph $D_{T}=$ $\left\{\left(i_{k}, i_{k-1}\right) \mid i \in\{2, \ldots, t\}\right\}$ with arcs going in the reverse direction of the order in the coalition. Let

\footnotetext{
${ }^{3}$ Every coalition having a unique largest feasible subset follows from the fact that $\Phi_{D}^{c}$ is union closed.

${ }^{4}$ For rooted trees, so also for line-graphs, the conjunctive approach coincides with the disjunctive approach to games with a permission structure where it is assumed that each player (except the top-players) needs permission from at least one of its predecessors, see Gilles and Owen (1999) and van den Brink (1997).

${ }^{5}$ Note the difference with null players in TU-games in characteristic function where all marginal contributions of a player being zero indeed implies that without this player the other players earn the same whether this player cooperates with them or not.
} 
$A=\left\{\mathbf{a}_{k}\right\}_{k \in\{1, \ldots, n\}}$ with $\mathbf{a}_{k} \in \mathbb{R}^{k}$ be such that its $l$-th component $\left(a_{k}\right)_{l} \geq 0$ for all $l \in\{1, \ldots, k\}$ and $\sum_{l=1}^{k}\left(a_{k}\right)_{l}=1$. To any ordered unanimity game $w_{T} \in \mathcal{G}^{N}$ we assign a coalitional (characteristic function) game $\hat{w}_{T}^{A} \in \mathcal{G}^{N}$ given by

$$
\hat{w}_{T}^{A}=\sum_{i \in H(T)}\left(a_{t}\right)_{i(T)} \hat{u}_{\{i\}}
$$

where $\hat{u}_{\{i\}}$ is the characteristic function of the (unordered) unanimity game on $\{i\}$. Then the corresponding solution $\psi^{A}$ on $\mathcal{G}^{N}$ is given by

$$
\psi^{A}(N, v)=\sum_{T \in \Omega(N)} \frac{\Delta_{v}^{*}(T)}{t !} \varphi\left(\hat{w}_{T}^{A}, D_{T}\right)
$$

with $\Delta_{v}^{*}(T)$ the dividends for generalized characteristic functions as defined in Sánchez and Bergantiños (1997), see Section 2. So, this solution assigns to every ordered coalition the permission value of a corresponding game with permission structure where the permission structure is a directed line-graph in the reverse order of the coalition, and the game is a convex combination of the unanimity games of the singletons in the coalition, i.e. a convex combination of additive games. This solution also can be written as

$$
\begin{aligned}
\psi_{i}^{A}(N, v) & =\sum_{T \in \Omega(N)} \frac{\Delta_{v}^{*}(T)}{t !} \varphi_{i}\left(\hat{w}_{T}^{A}, D_{T}\right) \\
& =\sum_{T \in \Omega(N)} \frac{\Delta_{v}^{*}(T)}{t !} \varphi_{i}\left(\sum_{j \in H(T)}\left(a_{t}\right)_{j(T)} \hat{u}_{\{j\}}, D_{T}\right) \\
& =\sum_{\substack{T \in \Omega(N), i \in H(T) \\
j \in H(T), j(T) \leq i(T)}} \frac{\left(a_{t}\right)_{j(T)}}{(t-j(T)+1) t !} \Delta_{v}^{*}(T) \text { for all } i \in N .
\end{aligned}
$$

Some special cases are the following:

1. If $\left(a_{k}\right)_{k}=1$ and $\left(a_{k}\right)_{l}=0$ for all $l \in\{1, \ldots, k-1\}$, then we obtain the NR-value $\psi^{N R}$.

2. If $\left(a_{k}\right)_{1}=1$ and $\left(a_{k}\right)_{l}=0$ for all $l \in\{2, \ldots k\}$, then we obtain the SB-value $\psi^{S B}$.

3. If $\left(a_{k}\right)_{l}=\frac{1}{k}$ for all $l \in\{1, \ldots, k\}$, then we obtain the linear solution determined by $\bar{\psi}\left(w_{T}\right)=$ $\frac{1}{n ! t} \sum_{j \in H(T)} P(j, T)$ with $P(j, T)_{i}=0$ if $i(T)<j(T)$ or $i \notin H(T)$, and $P(j, T)_{i}=\frac{1}{t-j(T)+1}$ if $i(T) \geq j(T)$.

Example 3.1 Consider the unanimity game $\left(N, w_{T}\right) \in \mathcal{G}^{N}$ with $N=\{1,2,3,4\}$ and $T=$ $(1,2,3)$. Then $D_{T}=\{(3,2),(2,1)\}$ and for all $A$ such that $\mathbf{a}_{3}=\left(\left(a_{3}\right)_{1},\left(a_{3}\right)_{2},\left(a_{3}\right)_{3}\right)$ we consider the coalitional (characteristic function) game $\hat{v}=\left(a_{3}\right)_{1} \hat{u}_{\{1\}}+\left(a_{3}\right)_{2} \hat{u}_{\{2\}}+\left(a_{3}\right)_{3} \hat{u}_{\{3\}}$.

1. If $\mathbf{a}_{3}=(0,0,1)$ then $\psi^{A}\left(N, w_{T}\right)=\frac{1}{6} \varphi\left(\hat{u}_{\{3\}}, D_{T}\right)=\frac{1}{6} \varphi^{S h}\left(N, \hat{u}_{\{3\}}\right)=\frac{1}{6}(0,0,1,0)=\psi^{N R}\left(N, w_{T}\right)$.

2. If $\mathbf{a}_{3}=(1,0,0)$ then $\psi^{A}\left(N, w_{T}\right)=\frac{1}{6} \varphi\left(\hat{u}_{\{1\}}, D_{T}\right)=\frac{1}{6} \varphi^{S h}\left(N, \hat{u}_{\{1,2,3\}}\right)=\frac{1}{6}\left(\frac{1}{3}, \frac{1}{3}, \frac{1}{3}, 0\right)=$ $\psi^{S B}\left(N, w_{T}\right)$. 
3. If $\mathbf{a}_{3}=\left(\frac{1}{3}, \frac{1}{3}, \frac{1}{3}\right)$ then $\psi^{A}\left(N, w_{T}\right)=\frac{1}{6} \varphi\left(\frac{1}{3}\left(\hat{u}_{\{1\}}+\hat{u}_{\{2\}}+\hat{u}_{\{3\}}\right), D_{T}\right)$

$=\frac{1}{6 \cdot 3}\left(\varphi^{S h}\left(N, \hat{u}_{\{1,2,3\}}\right)+\varphi^{S h}\left(N, \hat{u}_{\{2,3\}}\right)+\varphi^{S h}\left(N, \hat{u}_{\{3\}}\right)\right)=\frac{1}{18}\left(\left(\frac{1}{3}, \frac{1}{3}, \frac{1}{3}, 0\right)+\left(0, \frac{1}{2}, \frac{1}{2}, 0\right)+(0,0,1,0)\right)=$ $\frac{1}{18}\left(\frac{1}{3}, \frac{5}{6}, \frac{11}{6}, 0\right)=\frac{1}{108}(2,5,11)$.

Next we provide an axiomatic characterization of this class of solutions. Besides axioms that are already mentioned in Section 2, we use the following three. Although $\psi^{N R}$ and $\psi^{S B}$ are very different, they have in common that in unanimity games, players at the end of the unanimity coalition earn at least as much as players in the beginning of the unanimity coalition. In some sense, $\psi^{S B}$ is an extreme case where all players in the unanimity coalition get the same, while $\psi^{N R}$ is the other extreme case where all payoff goes to the last player. Although we consider these two solutions as extreme, we require from a solution that it satisfies this monotonicity with respect to the order of the players in the unanimity coalition. This will permit us to enlarge the set of solutions for generalized TU-games obtaining the class of order monotonic solutions. We will characterize this class, and in the next sections two particular subclasses.

Axiom 3.2 (Order monotonicity) If $T \in \Omega(N)$ and $i, j \in H(T)$ are such that $i(T)<j(T)$ then $\psi_{i}\left(N, w_{T}\right) \leq \psi_{j}\left(N, w_{T}\right)$.

The next axiom is a standard anonymity, meaning that the labels of players do not matter for their payoffs.

Axiom 3.3 (Anonymity) For every permutation $\pi: N \rightarrow N$ it holds that $\psi_{i}(N, v)=\psi_{\pi(i)}(N, \pi v)$.

Finally, we require that in monotone games all payoffs are nonnegative. A game $(N, v) \in \mathcal{G}$ is monotone, if $v(R) \leq v(S)$ whenever $R \widetilde{\subseteq} S$.

Axiom 3.4 (Nonnegativity) If $(N, v) \in \mathcal{G}$ is monotone, then $\psi_{i}(N, v) \geq 0$ for all $i \in N$.

It turns out that these three axioms, together with efficiency, linearity and the null player property (of Sánchez and Bergantiños (1997)) characterize the class of solutions defined above in (3.4). Therefore we refer to this class as the class of order monotonic solutions.

Theorem 3.5 A solution $\psi$ satisfies efficiency, linearity, the null player property, order monotonicity, anonymity and nonnegativity if and only if there is an $A=\left\{\mathbf{a}_{k}\right\}_{k \in\{1, \ldots, n\}}$, with $\mathbf{a}_{k} \in \mathbb{R}^{k}$ satisfying $\left(a_{k}\right)_{l} \geq 0$ for all $l \in\{1, \ldots, k\}$ and $\sum_{l=1}^{k}\left(a_{k}\right)_{l}=1$, such that $\psi=\psi^{A}$.

\section{PROOF}

It is straightforward to verify that all order monotonic solutions satisfy these properties. To show that only order monotonic solutions satisfy these properties, suppose that solution $\psi$ satisfies efficiency, linearity, the null player property, order monotonicity, anonymity and nonnegativity. We first prove uniqueness for unanimity games $\left(N, w_{T}\right)$ by induction on $t$. Therefore, first consider $w_{T}$ with $t=1$, i.e. $H(T)=\{j\}$. Since all $i \in N \backslash\{j\}$ are null players in $w_{T}$, the null player 
property implies that $\psi_{i}\left(N, w_{T}\right)=0$ for all $i \in N \backslash\{j\}$. Efficiency then requires that $\psi_{j}\left(N, w_{T}\right)=$ $\frac{1}{n !}$.

Proceeding by induction, suppose that there exists an $A=\left\{\mathbf{a}_{k}\right\}_{k \in\{1, \ldots, n\}}$, with $\mathbf{a}_{k} \in \mathbb{R}^{k}$ for all $k \in\{1, \ldots, n\}$, satisfying $\left(a_{k}\right)_{l} \geq 0$ for all $l \in\{1, \ldots, k\}$ and $\sum_{l=1}^{k}\left(a_{k}\right)_{l}=1$, such that $\psi\left(N, w_{T^{\prime}}\right)=$ $\psi^{A}\left(N, w_{T^{\prime}}\right)$ for all $T^{\prime} \in \Omega(N)$ with $t^{\prime}<t$, and consider $T \in \Omega(N)$ with $|H(T)|=t$. Since all $i \in N \backslash H(T)$ are null players in $\left(N, w_{T}\right)$, the null player property implies that $\psi_{i}\left(N, w_{T}\right)=0$ for all $i \in N \backslash H(T)$. Order monotonicity implies that $\psi_{i}\left(N, w_{T}\right) \leq \psi_{j}\left(N, w_{T}\right)$ for all $i, j \in H(T)$ with $i(T) \leq j(T)$. So, by efficiency, nonnegativity and order monotonicity there exist numbers $\lambda_{t, k} \geq 0, \lambda_{t, k} \geq \lambda_{t, k-1}$ for all $k \in\{2, \ldots, t\}$ and $\sum_{k=1}^{t} \lambda_{t, k}=1$ such that $\psi_{i}\left(N, w_{T}\right)=\frac{\lambda_{t, i(T)}}{n !}$ for all $i \in H(T)$. Taking $\left(a_{t}\right)_{1}=\lambda_{t, 1}$ and, recursively, $\left(a_{t}\right)_{k}=\lambda_{t, k}-\sum_{l=1}^{k-1}\left(a_{t}\right)_{l}$, we obtain that $\psi\left(N, w_{T}\right)=\psi^{A}\left(N, w_{T}\right)$.

Moreover, anonymity implies that $\psi_{i}\left(N, w_{T^{\prime}}\right)=\psi_{i}^{A}\left(N, w_{T^{\prime}}\right)=\lambda_{t, i\left(T^{\prime}\right)}$ for all $i \in H\left(T^{\prime}\right)$ whenever $t^{\prime}=t$ and $A=\left\{\mathbf{a}_{k}\right\}_{k \in\{1, \ldots, n\}}$ is as defined above.

Solution $\psi$ being equal to $\psi^{A}$ on the class of all generalized characteristic functions then follows from linearity of $\psi$ and (2.2).

Note that anonymity implies that $\psi_{i_{k}}\left(N, w_{\left\{i_{1}, \ldots, i_{t}\right\}}\right)=\psi_{i_{k}^{\prime}}\left(N, w_{\left\{i_{1}^{\prime}, \ldots, i_{t}^{\prime}\right\}}\right)$ for all $k \in\{1, \ldots, t\}$. The ratio $\frac{\left(a_{t}\right)_{k}}{\left(a_{t}\right)_{k+1}}$ can be different for different size $t$ as long as order monotonicity is satisfied.

Substituting in the previous theorem the order monotonicity axiom by the following axiom of rank monotonicity characterizes the so called arithmetic solution defined by:

$$
\psi_{i}^{a r t}(N, v)=\sum_{\substack{T \in \Omega(N) \\ i \in H(T)}} \Delta_{v}^{*}(T) \frac{2 i(T)}{(t+1) ! t} .
$$

Axiom 3.6 (Rank monotonicity) For every $N \subset \mathbb{N}$, there exist numbers $k_{t}, t \in\{1, \ldots n\}$, such that for every $T \in \Omega(N)$ and $i \in H(T)$ holds $\psi_{i}\left(N, w_{T}\right)=k_{t} i(T)$.

Theorem 3.7 Solution $\psi$ satisfies efficiency, linearity, the null player property, rank monotonicity, anonymity and nonnegativity if and only if it is the arithmetic solution $\psi^{\text {art }}$.

The obvious proof is omitted.

We argued before that we consider the symmetry axiom of Sánchez and Bergantiños (1997) to be too strong. A weaker symmetry requires equal payoffs for players that, besides being symmetric in the sense of Sánchez and Bergantiños (1997), also are such that for any ordered coalition containing both players, exchanging their position does not change the worth of the coalition. So, we call two players $i, j \in N$ fully symmetric in $v$ if they are symmetric in $v$ and, moreover, $v(T)=v\left(T_{i j}\right)$ for all $T \in \Omega(N)$ with $i, j \in H(T)$, where $T_{i j}$ is the ordered coalition (permutation of $T$ ) obtained by exchanging the positions of $i$ and $j$, i.e. $i\left(T_{i j}\right)=j(T), j\left(T_{i j}\right)=$ $i(T)$ and $h\left(T_{i j}\right)=h(T)$ for all $h \in H(T) \backslash\{i, j\}$.

Axiom 3.8 (Weak symmetry) If $i$ and $j$ are fully symmetric in $(N, v) \in \mathcal{G}$ then $\psi_{i}(N, v)=$ $\psi_{j}(N, v)$. 
All order monotonic solutions satisfy this weaker symmetry axiom which straightforward result we state without proof.

Proposition 3.9 Every order monotonic solution satisfies weak symmetry.

\section{The $p$-null player property and $p$-symmetry}

As mentioned before, the null player property of Nowak and Radzik (1994) ignores the role of players when players entering after them have a positive contribution but need those 'null players' to be present in order to make this contribution. In characterizing their solution this property implies that in the unanimity game on an ordered coalition $T=\left(i_{1}, \ldots, i_{t}\right)$, all players except player $i_{t}$ are null players. The null player property of Sánchez and Bergantiños (1997) is much weaker and also takes account of the role that players have in making players that enter after them productive, and thus none of the players in $H(T)$ is a null player in $\left(N, w_{T}\right)$.

As criticized before, the symmetry axiom of Sánchez and Bergantiños (1997) does not take account of possible asymmetries between players when they switch position in ordered coalitions they both belong to. Consequently, in the unanimity game on an ordered coalition $T=\left(i_{1}, \ldots, i_{t}\right)$, all players in $H(T)=\left\{i_{1}, \ldots, i_{t}\right\}$ are symmetric and earn the same payoff.

Both these approaches yield an order monotonic solution. They both can be seen as extreme cases, where the NR-value $\psi^{N R}$ only rewards the last player in every ordered unanimity coalition, while the SB-value $\psi^{S B}$ treats all equal. In this and the next sections we introduce classes of order monotonic solutions that contain the NR-value and SB-value as extreme cases. As explained above, a major difference between the NR- and SB-values is what they consider null players and symmetric players. The null player property of Nowak and Radzik (1994) is so extreme that they even do not need a symmetry axiom for their axiomatization. The null player property of Sánchez and Bergantiños (1997) is reasonable, but they need a strong symmetry axiom. Note that, under efficiency, the symmetry axiom of Sánchez and Bergantiños (1997) is incompatible with the null player property of Nowak and Radzik (1997) since according to the last axiom, in the unanimity game on ordered coalition $T$ all players except the last in order $T$ should get zero payoff, implying that the last player in $T$ should get all the payoff that is distributed according to efficiency, while according to the symmetry axiom of Sánchez and Bergantiños (1997) all players in $H(T)$ should get the same payoff. Next, we define compatible pairs of null player and symmetry axioms that, together with efficieny and linearity, characterize a solution.

Let $p \in[0,1]$. Player $i \in N$ is a $p$-null player in $(N, v) \in \mathcal{G}$ if for every ordered coalition $T=\left(i_{1}, \ldots i_{t}\right) \in \Omega(N \backslash\{i\})$, it holds that $v\left(T, i^{l}\right)=v(T)$ for all $l \in\{\lceil p(t+1)\rceil, \ldots, t+1\}$, where $\lceil z\rceil=\min \{x \in \mathbb{N} \mid z \leq x\}{ }^{6}$

Axiom 4.1 ( $p$-null player property) Let $p \in[0,1]$. If $i \in N$ is a $p$-null player in $(N, v) \in \mathcal{G}$ then $\psi_{i}(N, v)=0$.

\footnotetext{
${ }^{6}$ We consider $\mathbb{N}$ as the set of positive integers, so excluding 0 .
} 
For $p=1$, the 1-null player property only considers contributions to the last position and therefore coincides with the null player property of Nowak and Radzik (1994). For $p=0$, all positions are considered, and the 0-null player property is the null player property of Sánchez and Bergantiños (1997).

For $p \in[0,1]$, players $i, j \in N$ are $p$-symmetric in $(N, v) \in \mathcal{G}$ if for every ordered coalition $T \in \Omega(N \backslash\{i, j\})$, it holds that $v\left(T, i^{l}\right)=v\left(T, j^{l}\right)$ for all $l \in\{\lceil p(t+1)\rceil, \ldots, t+1\}$.

Axiom 4.2 (p-symmetry) Let $p \in[0,1]$. If $i, j \in N$ are $p$-symmetric in $(N, v) \in \mathcal{G}$ then $\psi_{i}(N, v)=\psi_{j}(N, v)$.

For $p=0,0$-symmetry compares the contributions of two players to any position in an ordered coalition, and equals the symmetry axiom of Sánchez and Bergantiños (1997). For $p=1$, it only compares contributions to the last position. Although 1-symmetry is satisfied by the NR-value, it is not necessary in the axiomatization in Theorem 2.5.

Theorem 4.3 Let $p \in[0,1]$. A solution $\psi$ satisfies efficiency, linearity, the $p$-null player property and p-symmetry if and only if it is $\psi^{A}$ given by (3.4) and (3.3) with $\left(a_{k}\right)_{l}=1$ if $l=\lceil p k\rceil$ and $\left(a_{k}\right)_{l}=0$ otherwise.

Proof

It is straightforward to verify that the solutions given in the theorem satisfy efficiency, linearity and the corresponding $p$-null player and $p$-symmetry axioms.

To show uniqueness, consider the generalized unanimity game $\left(N, w_{T}\right)$ with $T=\left(i_{1}, \ldots, i_{t}\right)$, and $p \in[0,1]$. By the $p$-null player property, $\psi_{i}\left(N, w_{T}\right)=0$ for all $i \in N \backslash\left\{i_{\lceil p t\rceil}, \ldots, i_{t}\right\}$. By $p$ symmetry all players in $\left\{i_{\lceil p t\rceil}, \ldots, i_{t}\right\}$ earn the same payoff, and thus the payoffs are determined by efficiency. Uniqueness on the class of all generalized characteristic functions then follows from linearity of $\psi$.

Note that $p=1$ yields the NR-value. The 1-null player property then only rewards the last player that enters in a unanimity coalition, and 1-symmetry only compares contributions to the last position. If $p=0$ we have the SB-value where the 0-null player property allows to reward all positions in a unanimity coalition, and symmetry requires that all players in the unanimity coaliton earn the same.

Example 4.4 Consider the unanimity game $\left(N, w_{T}\right)$ of Example 3.1. In Example 3.1 we already gave the NR-value $(p=1)$ and $S B$-value $(p=0)$. For $p=\frac{1}{2}$, i.e. $\lceil p t\rceil=\left\lceil\frac{3}{2}\right\rceil=2$, the solution yields $\psi^{\alpha}\left(N, w_{T}\right)=\frac{1}{6}\left(0, \frac{1}{2}, \frac{1}{2}, 0\right)$.

\section{A class of geometric solutions}

In del Pozo, Manuel, González-Arangüena and Owen (2011), the following parametric family of geometric solutions for generalized characteristic functions is introduced (and used to define 
centrality measures for directed networks). Given $\alpha \in[0,1]$, the solution $\psi^{\alpha}$ is given by

$$
\psi_{i}^{\alpha}(N, v)=\sum_{\substack{T \in \Omega(N) \\ i \in H(T)}} \frac{\alpha^{t-i(T)}}{t ! \sum_{l=0}^{t-1} \alpha^{l}} \Delta_{v}^{*}(T) \text { for all } i \in N .
$$

This class contains the NR-value $\psi^{N R}=\psi^{0}$ by taking $\alpha=0$, as well as the SB-value $\psi^{S B}=\psi^{1}$ by taking $\alpha=1$ as extreme cases. All these solutions are order monotonic since $\psi_{i(T)}^{\alpha}\left(N, w_{T}\right)=$ $\alpha \psi_{i(T)+1}^{\alpha}\left(N, w_{T}\right)$ for all $i \in H(T), T \in \Omega(N), i(T) \neq t$. In this section we provide axiomatizations for these geometric solutions.

We first generalize the enemy players property used in Manuel, González-Arangüena and van den Brink (2013), to characterize the Shapley value for classical TU-games to the context of generalized characteristic functions. We begin with defining what it means when players are indifferent to each other in a generalized characteristic function.

Definition 5.1 Given $(N, v) \in \mathcal{G}$, players $i, j \in N$ are related in $(N, v)$ if and only if $i=j$ or there exists a family of ordered coalitions in $\Omega(N),\left\{T_{l}\right\}_{l=1}^{m}$, verifying

i) $\Delta_{v}^{*}\left(T_{l}\right) \neq 0$ for all $l=1,2, \ldots, m$.

ii) $i \in H\left(T_{1}\right), j \in H\left(T_{m}\right)$ and $H\left(T_{l}\right) \cap H\left(T_{l+1}\right) \neq \emptyset$ for $l=1,2, \ldots, m-1$.

Player $i$ is indifferent to cooperate with player $j$ in $(N, v) \in \mathcal{G}$ if $i$ and $j$ are not related.

Similar as for classical TU-games, this property is symmetrical and we can say that $i$ and $j$ are indifferent to mutual cooperation, or indifferent players for short. We say that an ordered coalition is essential if it has a nonzero dividend. When two players decide not to cooperate (for instance, because they become enemies), the dividends $\Delta_{v}^{*}(T)$ of all ordered coalitions to which $i$ and $j$ both belong and enter consecutive and always in the same order (say $i$ before $j$ ), cannot be realized, and thus disappear when calculating the worth of these coalitions, yielding a transformation of the game. Note that for classical games we do not have the requirement that in every essential coalition the players enter consecutive and always in the same order, since for classical TU-games the order does not matter in the generation of worth. The idea behind two players becoming enemies in generalized characteristic functions is that it only affects their cooperation when they enter consecutive. When other players enter between them, then the fact that they become enemies is not effective. Also, the idea of players becoming enemies is asymmetric in the sense that only dividends dissappear when one of them enters (immediately) before the other.

We denote by $E(i, j)=\{T \in \Omega(N) \mid\{i, j\} \subseteq H(T)$ with $j(T)=i(T)+1\}$ the collection of all ordered coalitions to which $i$ and $j$ both belong and enter consecutive with $i$ entering first.

Definition 5.2 Given $(N, v) \in \mathcal{G}$ and $i, j \in N$ we define the game $\left(N, v^{i j}\right) \in \mathcal{G}$ as:

$$
v^{i j}=\sum_{T \in \Omega(N) \backslash E(i, j)} \Delta_{v}^{*}(T) w_{T} .
$$


Next, we generalize the enemy players property to generalized characteristic functions, stating that, when two players become enemies, meaning that the game $v^{i j}$ is played instead of game $v$, the effect on their respective payoffs is the same.

Axiom 5.3 A solution $\psi$ on $\mathcal{G}$ satisfies the enemy players property if, for all $(N, v) \in \mathcal{G}$ and every pair of players $i, j \in N$ which are not indifferent in generalized characteristic function $v$, it holds that: $\psi_{i}(N, v)-\psi_{i}\left(N, v^{i j}\right)=\psi_{j}(N, v)-\psi_{j}\left(N, v^{i j}\right)$.

Next, we generalize null contributions for indifferent players to the class of generalized characteristic functions, stating that, if one player $i$ is indifferent to cooperate with another player $j$, then he is even indifferent to the fact that this player leaves the game.

Axiom 5.4 A solution $\psi$ defined on $\mathcal{G}$ satisfies the null contributions for indifferent players property if, for all $(N, v) \in \mathcal{G}$ and every pair $i, j$ of indifferent players in generalized characteristic function $v$, it holds that: $\psi_{i}(N, v)-\psi_{i}\left(N \backslash\{j\},\left.v\right|_{N \backslash\{j\}}\right)=0$.

A solution $\psi$ on $\mathcal{G}$ satisfies the balanced contributions property of Sánchez and Bergantiños (1997) if, for all $(N, v) \in \mathcal{G}$ and all $i, j \in N, \psi_{i}(N, v)-\psi_{i}\left(\left.v\right|_{N \backslash\{j\}}\right)=\psi_{j}(N, v)-\psi_{j}\left(\left.v\right|_{N \backslash\{i\}}\right)$. This generalizes the balanced contributions property of Myerson (1980) for classical TU-games. The null contributions for indifferent players property establishes that, for indifferent players, the contributions must be balanced and, moreover, null. Therefore, in some sense it is more restrictive than balanced contributions of Sánchez and Bergantiños (1997) as it determines the value of the contributions. But in another sense it is less demanding because it only restricts the contributions for pairs of players that are indifferent. A similar remark was made in Manuel, Gonzalez-Arangüena and van den Brink (2013) for classical TU-games on Myerson (1980)'s balanced contributions.

Similar as for classical TU-games, by repeated application of the null contributions for indifferent players property, this property can be straightforward extended to the case in which a given player is indifferent to all the players in a group and so the proof is omitted.

Proposition 5.5 Let $(N, v) \in \mathcal{G}$ and $T \in \Omega(N)$. Suppose $i \in N \backslash H(T)$ is indifferent to each player in $H(T)$. If $\psi$ is a solution defined on $\mathcal{G}$ satisfying the null contributions for indifferent players property, then $\psi_{i}(N, v)=\psi_{i}\left(N \backslash H(T),\left.v\right|_{N \backslash H(T)}\right)$.

First, it turns out that these two axioms together with efficiency characterize the SB-value.

Theorem 5.6 A solution $\psi$ defined on $\mathcal{G}$ satisfies efficiency, the enemy players property and null contributions for indifferent players if and only if it is the $S B$-value $\psi^{S B}$.

Since this follows from the general Theorem 5.8, we postpone the proof.

Whereas the enemy players property seems reasonable for classical TU-games, for generalized characteristic functions on ordered coalitions, we might consider that also the positions of the players in the ordered coalitions have an effect on the change in their payoffs when they 
become enemies. Therefore, instead of having equal changes in payoff, we require that, when two players that are not indifferent become enemies, the effect on the payoff of the player who always enters first is some fraction $\alpha \in[0,1]$ of the change in payoff of the other.

Axiom 5.7 Let $\alpha \in[0,1]$. A solution $\psi$ on $\mathcal{G}$ satisfies the $\alpha$-enemy players property if, for all $(N, v) \in \mathcal{G}$ and every pair of players $i, j \in N$ which are not indifferent in $v$, it holds:

$$
\psi_{i}(N, v)-\psi_{i}\left(N, v^{i j}\right)=\alpha\left(\psi_{j}(N, v)-\psi_{j}\left(N, v^{i j}\right)\right) .
$$

Replacing the enemy players property in Theorem 5.6 by any $\alpha$-enemy players property, $\alpha \in(0,1]$, characterizes the corresponding geometric solution.

Theorem 5.8 Let $\alpha \in(0,1]$. A solution $\psi$ defined on $\mathcal{G}$ satisfies efficiency, the $\alpha$-enemy players property and null contributions for indifferent players if and only if it is the geometric solution $\psi^{\alpha}$.

\section{ProOF}

It is straightforward to verify that $\psi^{\alpha}, \alpha \in(0,1]$, satisfies efficiency, the $\alpha$-enemy players property and null contributions for indifferent players.

To prove uniqueness, take $\alpha \in(0,1]$ and consider a solution $\psi$ defined on $\mathcal{G}$ and satisfying efficiency, the $\alpha$-enemy players property and the null contributions for indifferent players property. We prove uniqueness by induction on the number of essential coalitions. Let $d(N, v)=|\delta(N, v)|$, where

$$
\delta(N, v)=\left\{T \in \Omega(N) \mid \Delta_{v}^{*}(T) \neq 0\right\} .
$$

If $d(N, v)=0$, then $(N, v)$ is the null game (i.e. $v(T)=0$ for all $T \in \Omega(N)$ ), and thus every $i \in N$ is indifferent to each player in $N \backslash\{i\}$. Therefore, by Proposition 5.5, $\psi_{i}(N, v)=\psi_{i}\left(\{i\}, v_{\mid\{i\}}\right)$ for all $i \in N$. As $v_{\mid\{i\}}(\{i\})=0$, by efficiency we have $\psi_{i}(N, v)=\psi_{i}\left(\{i\}, v_{\mid\{i\}}\right)=0$, and thus $\psi$ coincides with $\psi^{\alpha}$.

Suppose now, by the induction hypothesis, that $\psi(N, v)$ is uniquely determined for all games $\left(N, v^{\prime}\right) \in \mathcal{G}$ with $d\left(N, v^{\prime}\right)=k$, and consider a game $(N, v) \in \mathcal{G}$ with $d(N, v)=k+1$. Let $i \in N$ and let $C(i)$ be the set of players to whom $i$ is not indifferent. If $C(i)=\{i\}$, then similar as in the case $d(N, v)=0$ above, player $i$ is indifferent to each player in $N \backslash\{i\}$, and using the same reasoning as before, we have that $\psi_{i}(N, v)$ is uniquely determined.

Alternatively, suppose that $i \in N$ is such that $|C(i)|>1$, and let $j \in C(i), j \neq i$. By the definition of indifferent players, there exists a sequence of players $\left(i_{1}, i_{2}, \ldots, i_{r}\right)$ with $i_{1}=i, i_{r}=j$, $i_{l} \in C(i)$ for $l=1,2, \ldots, r$ and such that, for each $l=1,2, \ldots, r-1$, there is a $T_{l} \in \delta(N, v)$ with $\left\{i_{l}, i_{l+1}\right\} \subseteq H\left(T_{l}\right)$. Suppose that $T_{l}=\left(h_{1}, \ldots, h_{k}\right)$. As $\psi$ satisfies the $\alpha$-enemy players property, for every $p \in\{1, \ldots, k-1\}$ we have

$$
\psi_{h_{p}}(N, v)-\psi_{h_{p}}\left(N, v^{h_{p} h_{p+1}}\right)=\alpha\left(\psi_{h_{p+1}}(N, v)-\psi_{h_{p+1}}\left(N, v^{h_{p} h_{p+1}}\right)\right),
$$

and thus: 


$$
\psi_{h_{p}}(N, v)-\alpha \psi_{h_{p+1}}(N, v)=\psi_{h_{p}}\left(N, v^{h_{p} h_{p+1}}\right)-\alpha \psi_{h_{p+1}}\left(N, v^{h_{p} h_{p+1}}\right) .
$$

As $d\left(N, v^{h_{p} h_{p+1}}\right)<d(N, v)=k+1$, using the induction hypothesis $\psi_{h_{p}}\left(N, v^{h_{p} h_{p+1}}\right)$ and $\psi_{h_{p+1}}\left(N, v^{h_{p} h_{p+1}}\right)$ are determined and thus there exist known numbers $c^{h_{p} h_{p+1}}$ such that

$$
\psi_{h_{p}}(N, v)-\alpha \psi_{h_{p+1}}(N, v)=c^{h_{p} h_{p+1}} .
$$

Assuming, without loss of generality that $i_{l}=h_{q}$ and $i_{l+1}=h_{s}, q<s$, this yields that there exists a known number $\bar{c}^{i_{l}, i_{l+1}}$ such that

$$
\psi_{i_{l}}(N, v)-\alpha^{s-q} \psi_{i_{l+1}}(N, v)=\bar{c}^{i_{l}, i_{l+1}} .
$$

Since this holds for all $T_{l}$ in the sequence above, using this previous reasoning iteratively, there exist known numbers $\tilde{c}^{i j}>0$ and $\hat{c}^{i j}$ such that ${ }^{7}$

$$
\psi_{i}(N, v)-\tilde{c}^{i j} \psi_{j}(N, v)=\hat{c}^{i j} \text { for } j \in C(i)
$$

By the null contributions for indifferent players property:

$$
\psi_{j}(N, v)=\psi_{j}\left(C(i), v_{\mid C(i)}\right) \text { for all } j \in C(i),
$$

and thus:

$$
\sum_{j \in C(i)} \psi_{j}(N, v)=\sum_{j \in C(i)} \psi_{j}\left(C(i), v_{\mid C(i)}\right)
$$

where the right hand side is determined by efficiency. Therefore, we have $|C(i)|$ linear independent equations given by (5.6) and (5.7) in the $|C(i)|$ unknown payoffs $\psi_{j}(N, v), j \in C(i)$, which thus are uniquely determined.

Although the NR-value satisfies efficiency, the 0-enemy players property and null contributions for indifferent players, it is not characterized by these properties. However, strengthening null contributions for indifferent players to null contributions for weakly indifferent players, characterizes all geometric solutions $\psi^{\alpha}, \alpha \in[0,1]$, with the corresponding $\alpha$-enemy players property.

Definition 5.9 Given $(N, v) \in \mathcal{G}, i, j \in N$ are weakly indifferent in $(N, v)$ if and only if there is no ordered coalition $T$ with $\Delta_{v}^{*}(T) \neq 0$ and $\{i, j\} \subseteq H(T)$.

As this property is obviously symmetrical, whenever it holds, we say that $i$ and $j$ are weakly indifferent to mutual cooperation, or weakly indifferent players for short.

Axiom 5.10 A solution $\psi$ defined on $\mathcal{G}$ satisfies the null contributions for weakly indifferent players property if, for all $(N, v) \in \mathcal{G}$ and all pairs $i, j$ of weakly indifferent players in $(N, v)$, it holds that: $\psi_{i}(N, v)-\psi_{i}\left(\left.v\right|_{N \backslash\{j\}}\right)=0$.

\footnotetext{
${ }^{7}$ Note that $\tilde{c}^{i j}=\alpha^{z}$ for some $z \in \mathbb{N}$.
} 
Since two players that are indifferent (see Definition 5.1) are obviously weakly indifferent (see Definition 5.9), null contributions for weakly indifferent players implies null contributions for indifferent players.

Theorem 5.11 Let $\alpha \in[0,1]$. A solution $\psi$ defined on $\mathcal{G}$ satisfies efficiency, the $\alpha$-enemy players property and null contributions for weakly indifferent players if and only if it is the geometric solution $\psi^{\alpha}$.

\section{ProOF}

From Theorem 5.8 it follows that all $\psi^{\alpha}, \alpha \in(0,1]$ satisfy efficiency and the corresponding $\alpha$ enemy players property. For $\alpha=0$ this is obvious. It is also obvious that $\psi^{\alpha}$ satisfies null contributions for weakly indifferent players for all $\alpha \in[0,1]$.

Uniqueness for $\alpha \in(0,1]$ follows from Theorem 5.8 since null contributions for weakly indifferent players implies null contributions for indifferent players.

So, we are left to show uniqueness for the case $\alpha=0$. We again prove this by induction on the number of essential coalitions, but the proof goes different than the proof of uniqueness in Theorem 5.8. Consider a solution $\psi$ defined on $\mathcal{G}$ and satisfying efficiency, the 0 -enemy players property and the null contributions for weakly indifferent players property. Again, let $d(N, v)=|\delta(N, v)|$, where $\delta(N, v)=\left\{T \in \Omega(N) \mid \Delta_{v}^{*}(T) \neq 0\right\}$.

If $d(N, v)=0$, then $(N, v)$ is a null game (i.e. $v(T)=0$ for all $T \in \Omega(N)$ ), and thus every $i \in N$ is weakly indifferent to each player in $N \backslash\{i\}$. Therefore, similar as in the proof of Theorem 5.8, by Proposition 5.5, $\psi_{i}(N, v)=\psi_{i}\left(v_{\mid\{i\}}\right)$ for all $i \in N$. Again, $v_{\mid\{i\}}(\{i\})=0$ and by efficiency, $\psi_{i}(N, v)=\psi_{i}\left(\{i\}, v_{\mid\{i\}}\right)=0$, and thus $\psi$ coincides with $\psi^{0}=\psi^{N R}$.

Suppose now, by the induction hypothesis, that $\psi(N, v)$ is uniquely determined for games $\left(N, v^{\prime}\right) \in$ $\mathcal{G}$ with $d\left(N, v^{\prime}\right)=k$, and consider a game $(N, v) \in \mathcal{G}$ with $d(N, v)=k+1$. We distinguish three cases with respect to player $i \in N$.

First, if $C(i)=\{i\}$ (where $C(i)$ is again the set of players to whom $i$ is not indifferent), then similar as in the case $d(N, v)=0$ above, player $i$ is also weakly indifferent to each player in $N \backslash\{i\}$, and using the same reasoning as before, we have that $\psi_{i}(N, v)$ is uniquely determined.

Second $^{8}$, if there is a $T \in \delta(N, v)$ with $i \in T$ and $i(T) \neq t$ (so $i$ is not the last player in $T$ ) then the 0 -enemy players property implies that:

$$
\psi_{i}(N, v)-\psi_{i}\left(N, v^{i j}\right)=0
$$

where $j(T)=i(T)+1$. Since $d\left(N, v^{i j}\right)<k=d(N, v), \psi_{i}(N, v)=\psi_{i}\left(N, v^{i j}\right)$ is determined by the induction hypothesis.

Third, suppose that $T \in \delta(N, v)$ and $i \in T$ imply that $i(T)=t$, i.e. in every essential coalition player $i$ belongs to it has the last position. Suppose there are two players $i, j \in N, i \neq j$, that satisfy this condition. Then there is no $T \in \delta(N, v)$ such that $\{i, j\} \subseteq H(T)$ since they cannot both have the last position in $T$. Defining $W_{i}(N, v)=\{j \in N \mid\{i, j\} \subseteq H(T)$ for at least one

\footnotetext{
${ }^{8}$ From here the proof is different from that of Theorem 5.8.
} 
$T \in \delta(N, v)\}$, it holds that $\psi_{i}(N, v)=\psi_{i}\left(W_{i}(N, v),\left.v\right|_{W_{i}(N, v)}\right)$ by null contributions for weakly indifferent players. But since player $i$ is the only player in $\left(W_{i}(N, v),\left.v\right|_{W_{i}(N, v)}\right)$ for whom the payoff in $\left(W_{i}(N, v),\left.v\right|_{W_{i}(N, v)}\right)$ is not determined by the first or second case, the payoff of $i$ in $\left(W_{i}(N, v),\left.v\right|_{W_{i}(N, v)}\right)$ is uniquely determined by efficiency.

Since the NR-value and the SB-value are geometric solutions, we obtain axiomatizations of these solutions as a corollary.

Corollary 5.12 (i) A solution $\psi$ defined on $\mathcal{G}$ satisfies efficiency, the 0-enemy players property and null contributions for weakly indifferent players if and only if it is the NR-value $\psi^{N R}$.

(ii) A solution $\psi$ satisfies efficiency, the 1-enemy players property and null contributions for weakly indifferent players if and only if it is the $S B$-value $\psi^{S B} .9$

Note that for $\alpha=0$, the 0 -enemy players property is some kind of independence axiom stating that the payoff for the 'lower ordered' player does not change when two players become enemies. This is also the reason why in the proof of Theorem 5.11 we have to treat uniqueness for the case $\alpha=0$ separate.

We can make clear the distinction between players that are weakly indifferent and indifferent by using (undirected) graphs. An (undirected) graph on player set $N$ is a set $L \subseteq\{\{i, j\} \mid$ $i, j \in N, i \neq j\}$ of coalitions of size two. These elements are called edges or links. For game $(N, v) \in \mathcal{G}$ we can construct the graph $L^{v}=\{\{i, j\} \mid$ there is an ordered coalition $T \in \Omega(N)$ such that $\Delta_{v}^{*}(T) \neq 0$ and $\left.\{i, j\} \subseteq H(T)\right\}$ where two players are linked to each other if and only if they belong together to at least one ordered coalition that has a nonzero dividend. Then two players are weakly indifferent if they are not linked, while they are indifferent if they belong to different components or maximally connected coalitions, i.e. if there is no path connecting them.

\section{References}

Brânzei, R., V. Fragnelli, and S. Tijs (2002), Tree-connected peer group situations and peer group games. Mathematical Methods of Operations Research 55: 93-106.

Brink R van den (1997) An axiomatization of the disjunctive permission value for games with a permission structure. International Journal of Game Theory 26: 27-43.

Brink R van den, Gilles RP (1996) Axiomatizations of the conjunctive permission value for games with permission structures. Games and Economic Behavior 12: 113-126.

Gilles RP, Owen G (1999) Cooperative games and disjunctive permission structures. CentER Discussion Paper 9920, CentER for Economic Research, Tilburg University, Tilburg.

Gilles RP, Owen G, Brink R van den (1992) Games with permission structures: The conjunctive approach. International Journal of Game Theory 20: 277-293.

\footnotetext{
${ }^{9}$ This yields Theorem 5.6 as a Corollary.
} 
Gonzalez-Aranguena, E., C. Manuel, D. Gomez, and R. van den Brink (2008), "A value for directed communication situations", Tinbergen Discussion Paper 08/006-1, Tinbergen Institute and VU University, Amsterdam.

Harsanyi, J.C., 1959. A bargaining model for cooperative n-person games. In: Tucker, A.W., Luce, R.D. (Eds) Contributions to the Theory of Games IV, Princeton University Press, Princeton, pp 325-355.

Littlechild, S.C, and G. Owen (1973), "A simple expression for the Shapley value in a special case", Management Science, 20, 370-372.

Littlechild, S.C. and G.F. Thompson (1977), "Aircraft landing fees: A game theory approach", The Bell Journal of Economics, 8, 186-204.

Manuel, C., E. Gonzalez-Aranguena, E., and R. van den Brink (2013), "Players indifferent to cooperate and characterizations of the Shapley Value", Mathematical Methods of Operations Research, 77, 1-14.

Myerson RB (1977) Graphs and cooperation in games. Mathematics of Operations Research 2: $225-229$.

Myerson RB (1980) Conference structures and fair allocation rules. International Journal of Game Theory 9: 169-182.

Nowak AS, Radzik T (1994) The Shapley value for n-person games in generalized characteristic function form. Games and Economic Behavior 6: 150-161.

Pozo M del, Manuel C, González-Arangüena E, Owen G, (2011) Centrality in directed social networks. A game theoretic approach. Social Networks 33 : 191-200.

Sanchez E, Bergantiños G (1997) On values for generalized characteristic functions. OR Spectrum 19: 229-234.

Shapley LS (1953) A value for $n$-person games. In: Kuhn, H.W., Tucker, A.W. (Eds) Annals of Mathematics Studies, vol. 28. Princeton University Press, Princeton, NJ, pp. 307-317. 